\title{
Subacute rupture of a pseudoaneurysm formed by late rupture of a true left ventricular aneurysm
}

\author{
JOHN GOUDEVENOS, GARETH PARRY, GRAHAM N MORRITT \\ From the Regional Cardiothoracic Centre, Freeman Hospial, Newcastle upon Tyne
}

SUMMARY A man aged 34 in whom rupture of a true aneurysm at least four weeks after acute myocardial infarction led to the development of a pseudoaneurysm is described. Because the pseudoaneurysm ruptured subacutely and the haemodynamic, clinical, and echocardiographic signs were not consistent, diagnosis of the cardiac rupture was delayed. Operative repair was successful, but the patient died.

Left ventricular aneurysm is a common complication after myocardial infarction. Because false aneurysms (pseudoaneurysms) often rupture it is important to distinguish them from true aneurysms. ${ }^{1}$ Late rupture of a true aneurysm is unusual and subsequent formation of a pseudoaneurysm on its surface must be rare. We describe the diagnostic difficulties of this unusual complication of a myocardial infarction.

\section{Case report}

A 34 year old man was transferred from another hospital because of suspected cardiac rupture. Three months before he had suffered an acute anterior myocardial infarction from which he made an uneventful recovery. At that time haemoglobin was $171 \mathrm{~g} / \mathrm{l}$. Soon after infarction he completed a $7 \mathrm{~min}$ exercise test without symptoms but $2 \mathrm{~mm} S T$ depression was noted in lead III. He was discharged home and was treated with atenolol ( $50 \mathrm{mg}$ daily).

Four weeks later he was readmitted because of left sided chest pain, which was aggravated by deep inspiration and eased by sitting and leaning forward. His pulse rate was 65 beats per minute and blood pressure $100 / 60 \mathrm{~mm} \mathrm{Hg}$. A pericardial friction rub was heard. The haemoglobin concentration was $143 \mathrm{~g} / \mathrm{l}$, the white blood cell count was $9 \cdot 100 / \mathrm{mm}^{3}$, and the erythroctyte sedimentation rate $44 \mathrm{~mm} / \mathrm{h}$. Precordial $\mathrm{Q}$ waves consistent with the evolution of the anterior myocardial infarction were present on the electrocardiogram, which was essentially unchanged from the electrocardiogram on discharge.

Requests for reprints to Dr John Goudevenos, Cardiothoracic Centre, Freeman Hospital, Newcastle upon Tyne NE7 7DN.
There was no rise in the activity of cardiac enzymes. Cardiac antibodies were negative and a ventilationperfusion scan was normal. At a repeat test he exercised for $8 \mathrm{~min}$ with no ST segment depression. A gated isotope cardiac scan was consistent with an anterior aneurysm and poor left ventricular function (fig 1). A possible diagnosis of Dressler's syndrome was made and the patient was treated with aspirin and discharged home. The pain persisted in the left lower anterior chest. It varied in intensity and was aggravated by coughing and deep inspiration.

Two months after infarction the patient was readmitted because of severe retrosternal pain, which worsened with inspiration, and associated dyspnoea. The haemoglobin concentration was $134 \mathrm{~g} / \mathrm{l}$, the white blood count was $12 \cdot 600 / \mathrm{mm}^{3}$, and the erythrocyte sedimentation rate was $45 \mathrm{~mm} / \mathrm{h}$. The electrocardiogram and chest radiograph were unchanged. The activity of cardiac enzymes was normal. During a test he exercised for $8 \mathrm{~min}$ with no ST segment changes. He was discharged home on

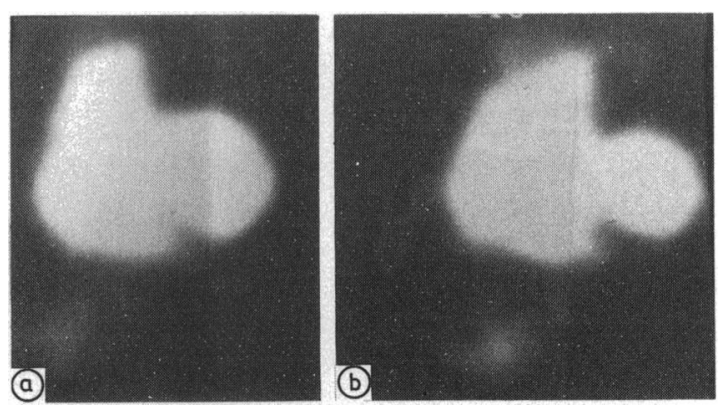

Fig 1 Gated pool scans four weeks after myocardial infarction during (a) systole and (b) diastole. A large left ventricular aneurysm is present. 
ibuprofen. He continued to experience pain at home.

Two days before he was transferred to our hospital sudden severe pain developed in the epigastrium and he collapsed with incontinence of faeces. He was admitted to the same hospital again. He was sweaty but there was no vomiting or melaena. The patient was pale, distressed, but conscious. His pulse rate was 116 per minute and blood pressure 100/ $60 \mathrm{~mm} \mathrm{Hg}$. The epigastrium was tender on palpation with guarding and rebound tenderness. An echocardiogram showed a moderate pericardial effusion and an apical ventricular aneurysm. Metabolic acidosis (lactate acid $8.7 \mathrm{mmol} / \mathrm{l}$ ) was found. The patient was transferred to this hospital the next day with a provisional diagnosis of a leaking ventricular aneurysm.

The patient smoked 20 cigarettes a day and weighed $95 \mathrm{~kg}$. There was no history of hypertension or diabetes. Examination showed a conscious, pale, unwell young man with poor peripheral perfusion. His temperature was $36.7^{\circ} \mathrm{C}$, pulse 100 per min, respiration rate 20 per min, and blood pressure $100 /$ $60 \mathrm{~mm} \mathrm{Hg}$ with a $15 \mathrm{~mm} \mathrm{Hg}$ paradox. Jugular venous pressure was raised $15 \mathrm{~cm}$ but Kussmaul's sign could not be detected. The heart was enlarged, with a diffuse apical impulse, and a third heart sound was present. A pericardial friction rub was audible. A few rales were audible in both lung bases. The epigastrium was tender and the bowel sounds quiet. The haemoglobin was $106 \mathrm{~g} / 1$ and the total serum creatine kinase was $149 \mathrm{IU} / 1$. The electrocardiogram showed signs of an old anterior myocardial infarction, without alteration in voltage. Chest $x$ ray showed car-
Goudevenos, Parry, Morritt $\frac{\text { D }}{\stackrel{一}{\Phi}}$ diomegaly. An echocardiogram showed pericardiak effusion with no signs of diastolic collapse of the right ventricle and an apical left ventricular aneurysm.

Cardiac catheterisation showed the following pres 는 sures: right atrium $26 / 18 \mathrm{~mm} \mathrm{Hg}$ (mean 24 ), righ를. ventricle $45 / 20 \mathrm{~mm} \mathrm{Hg}$; pulmonary artery $45 / \frac{\rho}{\sigma}$ $20 \mathrm{~mm} \mathrm{Hg}$ (mean 30), pulmonary artery wedge pres- $-\Omega$ sure $30 / 16 \mathrm{~mm} \mathrm{Hg}$ (mean 24), left ventricle 95/\% $22 \mathrm{~mm} \mathrm{Hg}$, and aorta $100 / 70 \mathrm{~mm} \mathrm{Hg}$.

There was a deep $x$ descent and a blunted y descent in the right atrial pressure waveform. A decrease in intra-aortic pressure of 10 to $15 \mathrm{~mm} \mathrm{Hg}$ during? inspiration was recorded. A coronary arteriogram? showed that the left anterior descending artery was totally occluded proximally. Left ventriculographyto showed a huge, true, anterior left ventricularit aneurysm (fig 2). There was a suggestion of anc opening in the apex of the true aneurysm (fig 2) but no evidence of contrast entering the pericardial space, and no definite conclusion about the cause of the tamponade could be reached. Pericardiocentesis? was attempted but was unsuccessful.

The patient was transferred to the operating theatre and sternotomy was performed. The pericar-dial cavity was full of old clot surrounding the heart ${ }_{00}^{\circ}$ A false aneurysm was present at the apex of the heart. $\subseteq$ This was firmly contained by the pericardium and ito was ruptured at one site. A large thrombus was present in the lumen of the true aneurysm. After removal of the thrombus the aneurysm was excised and patched under cardiopulmonary bypass. The patient died soon after the operation with signs of left ventricular failure.

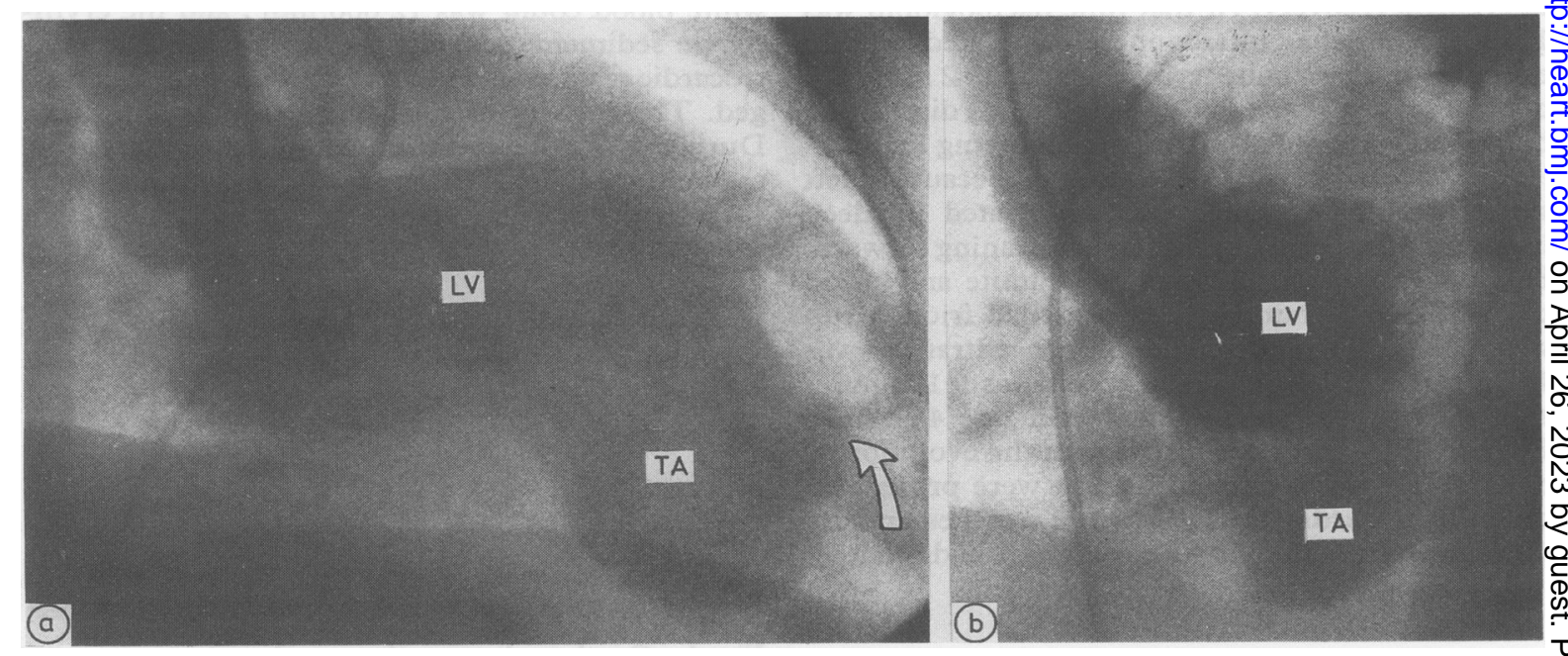

Fig 2 Left ventriculograms in (a) the right anterior oblique and (b) the left anterior oblique projections. LV, functioning left ventricle; $T A$, true aneurysm. The arrow indicates the site of the pseudoaneurysm. 
At necropsy the prosthetic patch was in position at the apex of the heart. The myocardium showed irregular fibrosis in the anterior wall of the left ventricle and the anterior half of the interventricular septum; the endocardium covering this area showed fibrous thickening. The histological appearance was consistent with this being an area of left ventricular infarction undergoing some resolution by fibrosis. The left anterior descending artery was occluded by old organised thrombus that completely filled the proximal $3 \mathrm{~cm}$ of the vessel. Recent, non-adherent thrombus was present in the left main artery extending to the vessel wall, and on cut section pale and dark areas were seen. The cause of death was believed to be left ventricular failure caused by peroperative embolisation of the left main stem and circumflex coronary arteries.

\section{Discussion}

Attention has been drawn to the difficulty in distinguishing between true and false ventricular aneurysms. ${ }^{1}$ Rupture of a left ventricular aneurysm is uncommon and occurs in the early stages of development. Late rupture, when the aneurysm has become stabilised by the formation of dense fibrous tissue in its wall, almost never occurs. ${ }^{2}$ We found only one report of a case of rupture of a true ventricular aneurysm at least a month after acute myocardial infarction. ${ }^{3}$

In contrast with true aneurysm, both large and small pseudoaneurysms may rupture during the early stages of development and also after the established fibrous stage is reached. ${ }^{2}$ The rupture may lead either to sudden fatal pericardial haemorrhage or to recurrent haemorrhagic effusion. ${ }^{4}$

The sequence of events in our patient is not clear. We believe that a true aneurysm developed after the acute myocardial infarction and was confirmed by gated pool scan four weeks later. At that stage the true aneurysm probably ruptured. Rupture was complicated by pericarditis and subsequent formation of the pseudoaneurysm. It is possible that there was a minor rupture of the pseudoaneurysm two months after the myocardial infarction and that this led to recurrent pericardial haemorrhage. But it is more likely that there was a major rupture of the pseudoaneurysm two months after its development (three months after the myocardial infarction) when the patient was admitted after collapse.

Our patient had classic haemodynamic findings of tamponade; but the classic clinical signs of tamponade, apart from raised venous pressure, were absent or were not pronounced. Echocardiography defined the true aneurysm but not the false aneurysm, and left ventriculography was suggestive of a false aneurysm. Good images were difficult to obtain in this obese patient. Despite the patient's continuing pain, the referring physicians were initially misled by the apparent well-being of the patient. London and London reported that repeated and prolonged chest pain was a feature in $55 \%$ of patients with cardiac rupture, ${ }^{5}$ possibly because of slow leakage of blood into the pericardial sac before clinically evident cardiac tamponade.

Although in most cases of cardiac rupture death is instantaneous, our patient survived at least 24 hours, long enough to reach the operating theatre. This outcome accords with the suggestion that cardiac rupture may be subacute and that when it is diagnosed during life it can be corrected surgically. ${ }^{6}$

We thank Dr D S Reid for his help in preparing this paper and for allowing us to report this case.

\section{References}

1 Davies MJ. Ischaemic ventricular aneurysms: true or false? Br Heart J 1988;60:95-7.

2 Vlodaver Z, Coe JI, Edwards JE. True and false ventricular aneurysms: propensity for the latter to rupture. Circulation 1975;51:567-72.

3 Cohen M, Packer M, Gorlin R. Indications for left ventricular aneurysmectomy. Circulation 1983;67: 717-22.

4 Cobel FL, Visudh-Arom K, Edwards JE. Pseudoaneurysm of the left ventricle leading to recurrent pericardial hemorrhage. Chest 1971;59:25-7.

5 London RE, London SB. Rupture of the heart. A critical analysis of 47 consecutive autopsy cases. Circulation 1965;31:202-5.

6 O'Rourke MF. Subacute heart rupture following myocardial infarction. Lancet 1973;ii:124-6. 